\title{
Rapid Molecular Diagnosis of Lactobacillus Bacteremia by Terminal Restriction Fragment Length Polymorphism Analysis of the 16S rRNA Gene
}

\author{
* Jeffrey E. Christensen, PhD, Clinical Research Center, Marshfield Clinic Research Foundation, Marshfield, Wisconsin \\ Cory E. Reynolds, MS, Clinical Research Center, Marshfield Clinic Research Foundation, Marshfield, Wisconsin \\ Sanjay K. Shukla, PhD, Clinical Research Center, Marshfield Clinic Research Foundation, Marshfield, Wisconsin \\ Kurt D. Reed, MD, Clinical Research Center, Marshfield Clinic Research Foundation, Marshfield, Wisconsin \\ ${ }^{*}$ Current affiliation: Department of Microbiology, University of Colorado Health Sciences Center, Denver, Colorado
}

\section{ABSTRACT}

\section{OBJECTIVE}

Bacteremia due to lactobacilli is uncommon, yet it is increasing in frequency, especially among immunosuppressed patients. In the clinical laboratory, lactobacilli must be subcultured from positive blood cultures before identification by traditional biochemical methods. Delays in diagnosis are significant because the organisms are inherently resistant to vancomycin, a drug frequently prescribed for empiric therapy for gram-positive bacteremia. Recently, we developed a rapid terminal-restriction fragment length polymorphism (T-RFLP) diagnostic assay based on species-specific variations in the bacterial 16S rRNA gene. We sought to apply this technique to the identification of Lactobacillus spp. from three cases of bacteremia.

DESIGN

The results of the T-RFLP analysis are compared with two standard biochemical identification methods.

METHODS

Lactobacillus strains were isolated from positive clinical blood cultures. Initial suspect cultures were subcultured and characterized using an automated substrate hydrolysis system and Lactobacillus carbohydrate fermentation profiles. Further biochemical and molecular analyses were performed from isolates propagated in Lactobacillus MRS broth. DNA was extracted and the 16S rRNA gene sequenced. Two sets of fluorescent labeled primers targeting the 16S rRNA gene were used for polymerase chain reaction (PCR) with chromosomal preparations from reference strains and blood isolates. The PCR products were digested with restriction enzymes and terminal-restriction fragment profile analysis performed.

\section{RESULTS}

T-RFLP analysis correctly identified the Lactobacillus species in each case. T-RFLP analysis could be completed within 8 hours of obtaining a positive blood culture as compared to more than the 24 to 48 hours required for traditional culturing and biochemical characterizations.

CONCLUSION

T-RFLP analysis allows for rapid identification of Lactobacillus directly from positive blood cultures and circumvents the requirement for subculture. Reduced diagnostic time has implications for duration of infection, the cost of patient care, length of hospitalization, development of broad-spectrum antibiotic resistance, and mortality due to bacteremia.

T-RFLP profiling represents a highly reproducible and predictive source for identification of many organisms associated with bacteremia.

RECEIVED: NOVEMBER 20, 2003

REPRINT REQUESTS:

Kurt D. Reed, MD

Clinical Research Center

Marshfield Clinic Research Foundation

1000 North Oak Avenue

Marshfield, WI 54449

Telephone: 715-389-5478

Fax: 715-389-3319

Email: reed.kurt@mcrf.mfldclin.edu
ACCEPTED: DECEMBER 1, 2003

\section{KEYWORDS:}

Lactobacillus; Bacteremia; PCR; 16S rRNA; Rapid diagnosis

GRANT SUPPORT:

Marshfield Clinic Research Foundation 


\section{INTRODUCTION}

The detection and identification of bacterial infections of the bloodstream is one of the most important functions of the clinical microbiology laboratory. Bacteremia results in significant morbidity and mortality, especially among patient populations that are immunocompromised..$^{1-3}$ One of the current limitations of processing blood cultures in the laboratory is the requirement to subculture isolates in order to perform biochemical or other tests needed for bacterial identification. This often results in one or more days of delay during which it is often necessary to administer broad-spectrum antimicrobial therapy. This practice contributes to the continued emergence of drug-resistant strains of pathogenic bacteria. In addition, some species of bacteria associated with bacteremia are inherently resistant to commonly prescribed antimicrobial agents and may not be effectively treated with empiric therapy. 4,5

Lactobacilli belong to a diverse group of bacteria that are gram-positive or gram-variable, facultatively anaerobic, nonspore forming, and produce lactic acid as a major product of carbohydrate fermentation. These bacteria are ubiquitous in the environment and propagate in ecological niches, such as plant surfaces and decaying plant material. In humans they colonize the oral cavity, gastrointestinal tract, and vagina. ${ }^{6}$ Lactobacillus casei appears to be the most frequently isolated species within the genus, ${ }^{7}$ although $L$. paracasei and $L$. rhamnosus are also encountered in clinical situations. However, isolates are often only identified by genus and most automated identification systems are not capable of accurate differentiation of Lactobacillus species. In general, lactobacilli are avirulent or of low virulence, but are recognized as opportunistic pathogens involved in bacteremia, ${ }^{8-11}$ as well as other significant infections such as amnionitis, ${ }^{12}$ abscesses, ${ }^{13}$ and endocarditis. ${ }^{7,9,14}$ Lactobacilli are also considered emerging pathogens in patients with chemotherapy induced neutropenia. ${ }^{15,16}$

One important clinical feature of lactobacilli is their inherent resistance to vancomycin, a drug frequently given as empiric therapy for bacteremia due to gram-positive bacteria. ${ }^{17}$ Therefore, rapid identification of lactobacilli from blood cultures would allow a more timely prescription of appropriate antimicrobial therapy. We recently described a rapid identification procedure for positive blood cultures that is based on terminal-restriction fragment length polymorphism (T-RFLP) profile analysis of the 16S rRNA gene. ${ }^{18}$ This molecular diagnostic procedure is applied directly to positive blood culture bottles and circumvents the need to subculture the isolate prior to identification. The assay is robust and is applicable to a broad range of bacterial pathogens. In this report we demonstrate the utility of T-RFLP in three cases of Lactobacillus bacteremia and compare the results with two conventional methods of bacterial identification.

\section{MATERIALS AND METHODS}

Bacterial strains and isolation

The Lactobacillus strains characterized in this study were isolated from positive blood cultures at Marshfield Laboratories as part of routine diagnostic testing. Marshfield Laboratories uses the BacT/Alert blood culturing system (Organon Teknika Corp., Durham, NC) and performs Gram staining for all positive blood cultures. Positive blood bottles were cultured on trypticase soy- $5 \%$ sheep blood or chocolate agar at $37^{\circ} \mathrm{C}$ under the appropriate atmospheric conditions. Blood bottle isolates were initially characterized using the automated VITEK system (bioMérieux Vitek Inc., Hazelwood, MO) and standard biochemical methods. Additional biochemical and molecular analyses described below were performed from isolates propagated in Lactobacillus MRS broth (Difco Laboratories, Detroit, MI).

\section{Anaerobe identification card}

The VITEK anaerobe identification card was used in conjunction with the VITEK system for the automated identification of each strain by characterizing the ability to hydrolyze 28 substrates. Following initial isolation and identification of each strain, cultures were propagated through MRS broth and plated on blood agar at $37^{\circ} \mathrm{C}$ under anaerobic conditions. Identification of isolates was repeated with the anaerobe identification cards as per the manufacturer's protocol.

\section{Lactobacillus carbohydrate fermentation profile}

The API $50 \mathrm{CH}$ test kit and the API CHL medium (bioMérieux Vitek Inc.) were used to determine the species of each Lactobacillus isolate by characterizing the ability to ferment 49 carbohydrates. Strains were grown for 18 hours to stationary phase in MRS broth and $1 \mathrm{~mL}$ of culture was harvested by centrifugation at $20,000 \mathrm{x}$ g for 1 minute. The cell pellets were washed with $1 \mathrm{~mL}$ sterile saline, suspended in $0.5 \mathrm{~mL}$ API CHL medium, and then further diluted 100fold in API CHL medium. The culture dilutions were then loaded to the API $50 \mathrm{CH}$ test strips as per the manufacturer's protocol, the capules were covered with mineral oil, and the test strips were incubated at $37^{\circ} \mathrm{C}$ for up to 48 hours. Test strips information was read and recorded at 16 and 40 hours. Carbohydrate fermentation profiling was performed in duplicate for each strain. Species identification was made by comparison of the results from API $50 \mathrm{CH}$ test strips with known carbohydrate fermentation profiles of lactobacilli as indicated in Bergey's Manual of Systematic Bacteriology. ${ }^{19}$

\section{DNA extraction}

DNA was extracted from cultured colonies using a modification of the tissue protocol for the QIAmp DNA Mini Kit (Qiagen, Inc., Valencia, CA). Briefly, $200 \mu \mathrm{L}$ of a cell suspension in $\mathrm{H}_{2} \mathrm{O}$ (containing $\sim 5$ to $10 \mathrm{mg}$ cells) was mixed with $200 \mu \mathrm{L}$ of QIAmp Buffer AL and $10 \mu \mathrm{L}$ of proteinase $\mathrm{K}(20 \mathrm{mg} / \mathrm{mL})$. Samples were incubated for 15 minutes at $56^{\circ} \mathrm{C}$ and then for 15 minutes at $95^{\circ} \mathrm{C}$. Two hundred microliters of ethanol was added and mixed by 
vortexing. The samples were loaded, washed, and eluted in $200 \mu \mathrm{L}$ from QIAmp spin columns, as indicated in the remainder of the manufacturer's protocol.

\section{Sequencing of the 16S rRNA gene}

The template DNA for sequencing of the 16S rRNA gene was amplified using primers FD1 (5'-AGA GTT TGA TCC TGG CTC AG) and RD1 (5'-AAG GAG GTG ATC CAG $\mathrm{CC})$, resulting in $\mathrm{PCR}$ products of $\sim 1500 \mathrm{bp}$. The reaction mixtures contained 1 x polymerase chain reaction (PCR) buffer, $200 \mu \mathrm{M}$ each deoxynucleoside triphosphate, $3.0 \mathrm{mM}$ $\mathrm{MgCl}_{2}, 0.5 \mu \mathrm{M}$ each primer species, 1.0 U Taq DNA polymerase, and $1.0 \mu \mathrm{L}$ chromosomal preparation per $15 \mu \mathrm{L}$ reaction. The PCR products were used for cycle sequencing reactions without further purification.

Cycle sequence reactions were prepared with BigDye Terminator mix (Applied Biosystems, Foster City, CA) as per supplier's protocol using primer FD1 (see above), RD1 (see above), 515F (5'-TGC CAG CAG CCG CGG TAA), 806R (5'-GGA CTA CCA GGG TAT CTA AT), 91E (5'-TCA AAK GAA TTG ACG GGG GC), or 13B (5'-AGG CCC GGG AAC GTA TTC AC). Sequence determination was done on an Applied Biosystems model 377XL automated DNA sequencing instrument. DNA sequences were aligned using Lasergene99 software (DNASTAR Inc., Madison, WI). The nucleotide sequences, determined from a minimum of 4-fold coverage, were deposited in GenBank under the accession numbers AY299486, AY299487, and AY299488. Sequence analysis was performed using the BLAST program for database searches at the National Center for Biotechnology Information Web site ${ }^{20}$ and the sequence match program at the Ribosomal Database Project (RDP-II) Web site. ${ }^{21}$ Multiple sequence alignments were performed using the ClustalW program. ${ }^{22}$

\section{PCR primers for determining T-RFLP}

Independent reactions with two sets of fluorescent labeled primers targeting the 16S rRNA gene were used for PCR with chromosomal preparations from reference strains and blood isolates. The primers were chosen as described in Christensen et al. ${ }^{18}$ based on evaluations of domain specific primers and maximization of matches received from queries using the TAP T-RFLP on-line analysis of RDP-II. As indicated, primers were labeled at the $5^{\prime}$ end with the dyes 6-carboxyfluorescein (6-FAM), 4,7,2',4',5',7'hexachloro-6-carboxyfluorescein (HEX), or N,N,N',N'tetramethyl-6-carboxyrhodamine (TAMRA).

The 5' region (encompassing hypervariable regions V1-V3) of the 16S rRNA gene was amplified with S-D-Bact-0045b-S-20 (5'-6-FAM-GCY TAA CAC ATG CAA GTY GA) and S-D-Bact-0785-a-A-19 (5'-HEX-CTA CCA GGG TAT CTA ATC C). The 3' region of the $16 \mathrm{~S}$ rRNA gene was amplified with S-D-Bact-0785-a-S-19 (5'-6-FAM-GGA TTA GAT ACC CTG GTA G) and S-D-Bact-1371-a-A-20 (5'-HEX-AGG CCC GGG AAC GTA TTC AC). PCR products from both sets of primers were predicted to be 600 $750 \mathrm{bp}$, with some variability dependent on the organism from which the template DNA is extracted.

\section{PCR conditions for T-RFLP}

Fluorescent dye labeled PCR products for T-RFLP were prepared as described previously. ${ }^{18}$ The reaction mixtures for PCR contained $1 \times$ PCR buffer, $200 \mu \mathrm{M}$ each deoxynucleoside triphosphate, $3.0 \mathrm{mM} \mathrm{MgCl}, 0.5 \mu \mathrm{M}$ each primer species, 1.0 U Taq DNA polymerase, and $1.0 \mu \mathrm{L}$ chromosomal preparation per $15 \mu \mathrm{L}$ reaction. Due to approximately 4-fold higher detection sensitivity of the fluorescence emission from the 6-FAM dye relative to HEX dye, a ratio of 1 part 6-FAM labeled primer $(0.125 \mu \mathrm{M})$ to 3 parts unlabeled primer $(0.375 \mu \mathrm{M})$ was used for primers $\mathrm{S}$ D-Bact-0045-b-S-20 and S-D-Bact-0785-a-S-19. DNA was amplified using a model 9600 thermal cycler (Perkin-Elmer, Norwalk, CT) with the following program: 15 minutes at $94^{\circ} \mathrm{C}$ for denaturation and $\mathrm{Taq}$ activation, 35 cycles consisting of denaturation ( 45 seconds at $94^{\circ} \mathrm{C}$ ), annealing (30 seconds at $52^{\circ} \mathrm{C}$ ), extension $\left(60\right.$ seconds at $\left.72^{\circ} \mathrm{C}\right)$, and a final extension for 5 minutes at $72^{\circ} \mathrm{C}$. All PCR reactions were analyzed by gel electrophoresis and stained with ethidium bromide to assure product formation prior to restriction digest.

\section{Restriction digests of PCR products}

PCR products were digested with restriction enzymes (Invitrogen, Carlsbad, CA and New England BioLabs, Beverly, MA) without further purification. Each $10 \mu \mathrm{L}$ restriction digest contained $1.0 \mu \mathrm{L}$ of PCR product, $0.5 \mu \mathrm{L}$ of restriction enzyme, and $8.5 \mu \mathrm{L}$ of prepared stock containing $2.5 \mathrm{ng} / \mu \mathrm{L}$ each appropriate size/digest control DNA in $1 \mathrm{x}$ buffer. The PCR product of 6-FAM labeled S-DBact-0045-b-S-20 and HEX labeled S-D-Bact-0785-a-A-19 (amplifying the 5' region of the 16S rRNA gene) was digested in separate reactions with $A l u \mathrm{I}, H h a \mathrm{I}, M s p \mathrm{I}$, and $R s a$ I. The PCR product of 6-FAM labeled S-D-Bact-0785-aS-19 and HEX labeled S-D-Bact-1371-a-A-20 (amplifying the 3 ' region of the $16 \mathrm{~S}$ rRNA gene) was only digested with HhaI. Restriction digests were performed in a model 9600 Perkin-Elmer thermal cycler programmed for a 1 hour incubation at $37^{\circ} \mathrm{C}$ followed by 20 minutes at $65^{\circ} \mathrm{C}$ for enzyme inactivation. The restriction digest products were purified with DyeEx 96-well spin columns (Qiagen) as per manufacturer's protocol.

\section{Terminal restriction fragment (TRF) profile analysis and organism identification}

Samples were prepared for electrophoresis by combining 1.0 $\mu \mathrm{L}$ of restriction digest product, $0.50 \mu \mathrm{L}$ of X-rhodamine MapMarker 1000 XL (BioVenture, Inc., Muffreesboro, TN), and $14 \mu \mathrm{L}$ of Hi-Di formamide (Applied Biosystems Instruments). The size standard contains single strands of DNA with a single ROX fluorophore at 50, 75, 100, 125, 150, 200, 250, 300, 350, 400, 450, 475, 500, 550, 600, 650, $700,750,800,850,900,950$, and 1000 bases. Samples were mixed by pipetting, denatured at $94^{\circ} \mathrm{C}$, and immediately 
cooled to $4^{\circ} \mathrm{C}$. The lengths of TRFs derived from amplified DNA products were determined with an Applied Biosystems model 3100 capillary DNA sequencing instrument operating in GeneScan ${ }^{\mathrm{TM}}$ mode. The samples were loaded to a $50 \mathrm{~cm}$ capillary with an injection voltage of $1 \mathrm{kV}$ for 22 seconds and run at $12.2 \mathrm{kV}$ for 2.2 hours at $50^{\circ} \mathrm{C}$. The sizes of control and sample TRFs were calculated by comparison with the internal standard using the local Southern method. ${ }^{23}$ Fragment lengths $\geq 20$ but $<50$ bases were calculated by linear extrapolation from the migration times of the 50 and 75 base standards.
Identification of bacteria was accomplished through the use of a program for calculating terminal fragment sizes and a searchable terminal restriction fragment profile database (TRFPD). ${ }^{18}$ Briefly, the maximum area 6-FAM and HEX peak data for each sample are identified and organized according to the respective primer set and restriction digest combination. The program is then used to compare the sample TRF profile with 2435 TRF profiles representing 921 different species of potential relevance to bacteremia in the TRFPD. The results from a Gram stain can also be selected in the data input window to limit the search. The

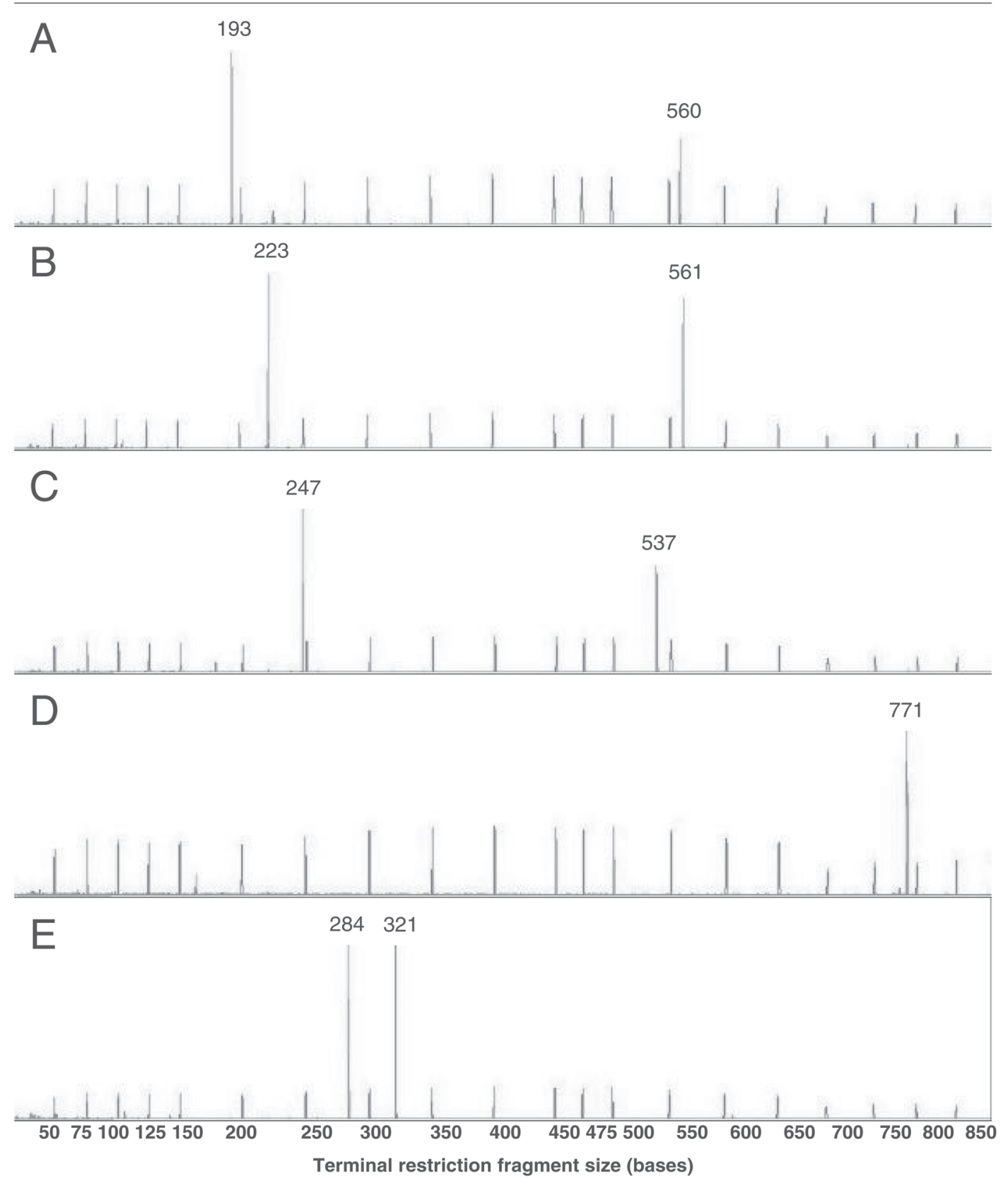

Figure 1. Representative TRF profile of the PCR products from the 16S rRNA gene of Lactobacillus rhamnosus MCRF-412. Each panel shows a PCR product/restriction digest electropherogram and the applicable range of the standard curve (50-850 bases). The single major 6-FAM (blue peaks) and HEX (green peaks) fragment of each digest are identified by their respective nucleotide size. TRFs from PCR products obtained with (6-FAM)-S-D-Bact-0045-b-S-20 and (HEX)-S-D-Bact-0785-a-A-19 are shown in panels A (Alul), B (Hhal), C (Mspl), and D (Rsal). TRFs from PCR products obtained with (6-FAM)-S-D-Bact-0785-aS-19 and (HEX)-S-D-Bact-1371-a-A-20 are shown in panel E (Hhal). The presence of a single peak size $>700$ bases for both the 6-FAM and HEX fragments in panel D indicates the PCR product was not digested with Rsal. 
Table 1. VITEK anaerobe identification.

\begin{tabular}{|c|c|c|}
\hline \multirow[b]{2}{*}{ Substrate } & \multicolumn{2}{|c|}{ Isolate, substrate hydrolysis, and identification ${ }^{a}$} \\
\hline & MCRF-271 \& MCRF-412 & MCRF-284 \\
\hline$\rho$-nitrophenyl phosphate & - & - \\
\hline$\rho$-nitrophenyl phosphate choline & - & - \\
\hline$\rho$-nitrophenyl- $\beta$-D-galactopyranoside & Positive & Positive \\
\hline$\rho$-nitrophenyl- $\alpha$-D-galactopyranoside & - & - \\
\hline$\rho$-nitrophenyl- $\beta$-D-glucopyranoside & Positive & Positive \\
\hline$\rho$-nitrophenyl- $\alpha$-D-glucopyranoside & Positive & Positive \\
\hline$\rho$-nitrophenyl- $\beta$-D-glucuronide & - & - \\
\hline$\rho$-nitrophenyl- $\beta$-D-lactoside & - & - \\
\hline$\rho$-nitrophenyl- $\alpha$-D-mannopyranoside & Weak & - \\
\hline$\rho$-nitrophenyl- $\alpha$-L-fucopyranoside & - & - \\
\hline$\rho$-nitrophenyl- $\beta$-D-fucopyranoside & - & - \\
\hline$\rho$-nitrophenyl- $\beta$-D-xylopyranoside & - & - \\
\hline$\rho$-nitrophenyl- $\alpha$-L-arabinofuranoside & - & - \\
\hline$\rho$-nitrophenyl- $\mathrm{N}$-acetylglucosaminide & Positive & Positive \\
\hline $\mathrm{N}$-benzoyl-DL-arginine $\rho$-nitroanilide & - & - \\
\hline L-leucine $\rho$-nitroanilide & Positive & Positive \\
\hline L-proline $\rho$-nitroanilide & - & - \\
\hline L-alanine $\rho$-nitroanilide & Positive & Positive \\
\hline L-lysine $\rho$-nitroanilide & Positive & Positive \\
\hline$\gamma$-glutamyl $\rho$-nitroanilide & - & - \\
\hline Triphenyl tetrazolium & - & - \\
\hline Arginine & - & - \\
\hline Urea & - & - \\
\hline Glucose & Positive & Positive \\
\hline Trehalose & Positive & Positive \\
\hline Arabinose & - & - \\
\hline Raffinose & - & - \\
\hline Xylose & - & - \\
\hline VITEK Identification & L. casei & L. casei \\
\hline
\end{tabular}

${ }^{a}$ Symbols for MCRF isolate results from the VITEK (VITEK (bioMérieux Vitek Inc., Hazelwood, MO) anaerobe identification: positive, positive for hydrolysis; weak, limited for hydrolysis; -, no detectable hydrolysis.

result of a search includes the number of matching fragment lengths (within chosen bp windows), ordering of closest matches, and links to best match TRF profiles. In addition, a sum of differences (SOD) score was determined for each TRF profile relative to the predicted fragments from the database. Specifically, the difference between the size of the experimentally determined TRF and the sequence predicted fragment size was calculated for each fragment/digest combination, and then the sum of these differences was calculated. A smaller SOD score is taken to represent a closer match to a given organism in the database.

\section{RESULTS}

Characterization of organisms from positive blood bottles The three organisms described in this manuscript were isolated from positive anaerobic blood bottles in Marshfield Laboratories. Each culture was determined to contain either Gram positive or Gram variable rod-shaped cells. Isolates grew in 24 hours on trypticase soy-5\% sheep blood or chocolate agar at $37^{\circ} \mathrm{C}$ under anaerobic conditions. Isolates were used for the identification of each organism using the following biochemical tests. 
Table 2. Identification by carbohydrate fermentation profile.

\begin{tabular}{|c|c|c|c|c|}
\hline Carbohydrate & L. rhamnosus ${ }^{\mathrm{a}}$ & $\begin{array}{c}\text { MCRF-271 \& } \\
\text { MCRF-412 }^{\mathrm{b}} \\
\end{array}$ & L. case $^{\mathrm{a}}$ & MCRF-284 \\
\hline Amygdaline & Positive & Positive & Positive & Positive \\
\hline D-arabinose & C & Weak & - & - \\
\hline Cellobiose & Positive & Positive & Positive & Positive \\
\hline Esculine & Positive & - & Positive & - \\
\hline D-fructose & Positive & Positive & Positive & Positive \\
\hline Galactose & Positive & Positive & Positive & Positive \\
\hline D-glucose & Positive & Positive & Positive & Positive \\
\hline Gluconate & Positive & Weak & Positive & Weak \\
\hline Lactose & Positive & Positive & C & Positive \\
\hline Maltose & Positive & - & Positive & Positive \\
\hline Mannitol & Positive & Positive & Positive & Positive \\
\hline D-mannose & Positive & Positive & Positive & Positive \\
\hline Melezitose & Positive & Positive & Positive & Positive \\
\hline Melibiose & - & - & - & - \\
\hline D-raffinose & - & - & - & - \\
\hline Rhamnose & Positive & Positive & - & - \\
\hline Ribose & Positive & Positive & Positive & Positive \\
\hline Salicine & Positive & Positive & Positive & Positive \\
\hline Sorbitol & Positive & Positive & Positive & Positive \\
\hline Trehalose & Positive & Positive & Positive & Positive \\
\hline D-xylose & - & - & - & - \\
\hline L-xylose & - & - & - & - \\
\hline
\end{tabular}

${ }^{a}$ Symbols for indicated reference organism profile from Bergey's Manual ${ }^{19}$ : positive, $90 \%$ or more strains positive for fermentation of the indicated carbohydrate; -, $90 \%$ or more strains negative; C, $11-89 \%$ strains positive.

${ }^{\mathrm{b}}$ Symbols for MCRF isolate results using the API $50 \mathrm{CH}$ source strips: positive, positive for fermentation of indicated carbohydrate; weak, limited for fermentation; -, no detectable fermentation.

VITEK anaerobe identification

The substrate hydrolysis results from the anaerobe identification card are shown in table 1 . The single difference found between the strains was the inability of MCRF-284 to use $\rho$-nitrophenyl- $\alpha$-D-mannopyranoside, whereas strains MCRF-271 and MCRF-412 provided a weak reaction. All three strains were identified as $L$. casei on the basis of the results from Gram reaction, lack of indole production, and substrate hydrolysis on the anaerobic identification card.

\section{Identification of Lactobacillus species by carbohydrate} fermentation profile

Table 2 shows the API $50 \mathrm{CH}$ results and the predicted carbohydrate fermentation profiles for $L$. casei and $L$. rhamnosus with 22 carbohydrates necessary for species differentiation and identification of most heterofermentative lactobacilli. Strains of L. casei and L. rhamnosus were differentiated by the ability of the latter isolates to ferment arabinose and rhamnose. In addition to the data shown in table 2, all of the blood isolates were positive for fermentation of arbutine, $\beta$-gentiobiose, D-tagatose, inositol, $\mathrm{N}$-acetyl-glucosamine, and saccharose. Also, strains MCRF271 and MCRF-412 were positive for fermentation of L-fucose and L-sorbose, while no reaction was found with MCRF-284. Conversely, MCRF-284 was positive for fermentation of adonitol, D-turanose, and inuline, while no reaction was found with MCRF-271 and MCRF-412.

Identification of species by sequence determination of 16S rRNA gene

The nucleotide sequence was determined for a 1480 base region of the $16 \mathrm{~S}$ rRNA gene from each of the blood isolates (GenBank accession numbers AY299486, AY299487, and AY299488). The sequences determined for MCRF-271 and MCRF-412 were identical for all 1480 bases. The BLAST analysis of MCRF-271 and MCRF-412 nucleotide sequence indicated the highest identities with a L. casei $(99.5 \%)$ and L. rhamnosus $(99.2 \%)$ strain. The similarity scores from the 
Table 3. TRF profiles for blood isolates and SOD scores for TRF database matches.

\begin{tabular}{|c|c|c|c|c|c|c|c|c|c|c|c|c|}
\hline \multirow{3}{*}{ Isolate or organisma } & \multirow{3}{*}{ Strain } & \multicolumn{10}{|c|}{ Size (bases) of TRF obtained with: } & \multirow{3}{*}{$\begin{array}{l}\text { Sum of differences } \\
\text { (SOD) score }\end{array}$} \\
\hline & & \multicolumn{2}{|c|}{ Alu $I^{b}$} & \multicolumn{2}{|c|}{ Hha $I^{b}$} & \multicolumn{2}{|c|}{ Msp I ${ }^{\mathbf{b}}$} & \multicolumn{2}{|c|}{ Rsa I $\mathbf{I}^{\mathbf{b}}$} & \multicolumn{2}{|c|}{ Hha Ic } & \\
\hline & & 6-FAM & HEX & 6-FAM & HEX & 6-FAM & HEX & 6-FAM & HEX & 6-FAM & HEX & \\
\hline MCRF-271 / MCRF-412 & & 193 & 560 & 561 & 223 & 537 & 247 & UD & UD & 321 & 284 & NA \\
\hline L. rhamnosus & DSM 20021 & 194 & 556 & 561 & 224 & 535 & 246 & UD & UD & 325 & 283 & 14 \\
\hline L. rhamnosus & JCM 1136 & 194 & 555 & 560 & 224 & 534 & 246 & UD & UD & 325 & 283 & 17 \\
\hline L. plantarum & NCDO 1752 & 175 & 558 & 562 & 224 & 536 & 246 & UD & UD & 320 & 283 & 26 \\
\hline L. plantarum & JCM 1149 & 175 & 556 & 560 & 224 & 534 & 246 & UD & UD & 325 & 283 & 33 \\
\hline L. plantarum & ATCC 8014 & 175 & 556 & 560 & 224 & 534 & 246 & UD & UD & 325 & 283 & 33 \\
\hline L. manihotivorans & LMG 18010 & 174 & 556 & 560 & 224 & 534 & 246 & UD & UD & 325 & 283 & 34 \\
\hline L. pentosus & JCM 1588 & 226 & 556 & 560 & 224 & 534 & 246 & UD & UD & 325 & 283 & 48 \\
\hline L. casei & ATCC 334 & 194 & 556 & 561 & 172 & 535 & 246 & UD & UD & 325 & 283 & 64 \\
\hline L. casei & ATCC 393 & 194 & 556 & 561 & 172 & 535 & 246 & UD & UD & 325 & 283 & 64 \\
\hline L. zeae & ATCC 15820 & 194 & 556 & 561 & 172 & 535 & 246 & UD & UD & 325 & 283 & 64 \\
\hline L. casei & JCM 1134 & 195 & 555 & 561 & 172 & 535 & 246 & UD & UD & 325 & 283 & 66 \\
\hline L. paracasei & JCM 1133 & 194 & 555 & 560 & 172 & 534 & 246 & UD & UD & 325 & 283 & 67 \\
\hline L. paracasei & JCM 8130 & 194 & 555 & 560 & 172 & 534 & 246 & UD & UD & 325 & 283 & 67 \\
\hline L. paracasei & JCM 1171 & 194 & 555 & 560 & 172 & 534 & 246 & UD & UD & 325 & 283 & 67 \\
\hline MCRF-284 & & 193 & 560 & 561 & 172 & 537 & 247 & UD & UD & 321 & 284 & NA \\
\hline L. casei & АTCC 334 & 194 & 556 & 561 & 172 & 535 & 246 & UD & UD & 325 & 283 & 13 \\
\hline L. casei & ATCC 393 & 194 & 556 & 561 & 172 & 535 & 246 & UD & UD & 325 & 283 & 13 \\
\hline L. zeae & ATCC 15820 & 194 & 556 & 561 & 172 & 535 & 246 & UD & UD & 325 & 283 & 13 \\
\hline L. casei & JCM 1134 & 195 & 555 & 561 & 172 & 535 & 246 & UD & UD & 325 & 283 & 15 \\
\hline L. paracasei & JCM 1133 & 194 & 555 & 560 & 172 & 534 & 246 & UD & UD & 325 & 283 & 16 \\
\hline L. paracasei & JCM 8130 & 194 & 555 & 560 & 172 & 534 & 246 & UD & UD & 325 & 283 & 16 \\
\hline L. paracasei & JCM 1171 & 194 & 555 & 560 & 172 & 534 & 246 & UD & UD & 325 & 283 & 16 \\
\hline L. rhamnosus & DSM 20021 & 194 & 556 & 561 & 224 & 535 & 246 & UD & UD & 325 & 283 & 65 \\
\hline L. paracasei & JCM 1181 & 194 & 555 & 612 & 172 & 534 & 246 & UD & UD & 325 & 283 & 66 \\
\hline L. rhamnosus & JCM 1136 & 194 & 555 & 560 & 224 & 534 & 246 & UD & UD & 325 & 283 & 68 \\
\hline
\end{tabular}

UD: Undigested PCR product (6-FAM and HEX fragment sizes were both $>700$ bases).

${ }^{\mathrm{a}}$ Organisms are presented in ascending order of the SOD scores relative to the TRF results for each MCRF strain.

'Labeled fragments derived from the PCR product of (6-FAM)-S-D-Bact-0045-b-S-20 and (HEX)-S-D-Bact-0785-a-A-19 or as predicted from sequence data.

'Labeled fragments derived from the PCR product of (6-FAM)-S-D-Bact-0785-a-S-19 and (HEX)-S-D-Bact-1371-a-A-20 or as predicted from sequence data.

${ }^{d}$ Value calculated from the differences between the size of the experimentally determined TRF and the sequence predicted fragment sizes for a restriction digest of

organisms in the TRF database. The SOD for each organism was then calculated.

${ }^{e}$ Each TRF size reported was determined from the average of at least two digests.

RDP-II sequence match analysis of MCRF-271 and MCRF-412 were determined to be 0.991 for $L$. casei and 0.986 for L. rhamnosus.

The BLAST analysis of MCRF-284 nucleotide sequence indicated the highest identities with two L. casei and one L. paracasei strains (100\% matches). The RDP-II similarity score obtained with the MCRF-284 sequence was determined to be 1.000 with two L. casei and one L. paracasei strains.

\section{Identification of species by TRF profile}

The TRF profiles obtained from restriction digest of the $16 \mathrm{~S}$ rRNA gene PCR products for each of the blood isolates matched closely to those predicted from their respective nucleotide sequences (figure 1 and table 3 ). The TRF profiles for MCRF-271 and MCRF-412 were closest to those for both L. rhamnosus strains in the sequence predicted TRFPD, matching all 10 fragments within 5 bases and having a SOD score of 14 and 17 bases (table 3 ). The next closest matches were for three strains of L. plantarum, but the profiles matched only 9 of 10 fragments within 5 bases and had a higher SOD score (26-33 bases). The latter results were primarily due to the significant differences in the 6-FAM labeled AluI restriction digest product (18 base difference).
The TRF profile for MCRF-284 was closest to those for three L. casei strains and one L. zeae strain in the TRFPD, matching all 10 fragments within 5 bases for all four strains and having a SOD score of 13-15 bases. The next closest matches were for three strains of $L$. paracasei, again matching all 10 fragments within 5 bases, but with a slightly greater SOD score (16 base difference).

\section{DISCUSSION}

This study describes the use of TRF profiling for identification of Lactobacillus species from blood cultures and comparison of this technique with other methods of identification. In a previous study, ${ }^{18}$ we showed that TRF profiling was effective in differentiating many organisms responsible for bacteremia.

Biochemical characterization of the blood culture isolates using the VITEK anaerobe system resulted in identification of $L$. case $i$ for all three strains, although the substrates available in the panel tests are not expected to differentiate all Lactobacillus species. To attempt more accurate identification of the blood culture isolates we tested their ability to ferment 49 carbohydrates in a commercially available kit for Lactobacillus speciation, which resulted in differentiation of the strains to L. rhamnosus and L. casei (table 2). This was primarily due to the ability of the L. rhamnosus strains 
to ferment rhamnose and D-arabinose. Although the lack of differentiation of these strains using the VITEK anaerobe system is likely of limited clinical significance, the primary disadvantage of both biochemical identification systems is the requirement for isolated colonies to be grown out from the positive blood culture bottles before analysis.

The analysis of DNA sequence data from the 16S rRNA gene of the blood isolates clearly indicated all three strains were lactobacilli. However, differentiation of species was less obvious due to the high degree of BLAST identity scores and RDP-II similarity scores between the 16S rRNA sequences of some $L$. casei and $L$. paracasei strains, and also between some L. rhamnosus and $L$. casei strains. Since Genbank is a public database, there may be some ambiguity as a result of phenotypic misidentification of the Lactobacillus species for which 16S rRNA gene sequence have been submitted.

The blood culture isolates were clearly identified as lactobacilli using TRF profiling. The MCRF-271 and MCRF-412 TRF profile had a conclusive SOD score that identified them as L. rhamnosus. However, similar to identification by $16 \mathrm{~S}$ rRNA gene sequencing, determination of species for MCRF-284 was not as obvious. The MCRF-284 TRF profile had similar SOD scores with $L$. casei and $L$. zeae, with a slightly greater difference from $L$. paracasei.

Commercially available biochemical identification systems often require isolation of a microorganism after growth in a blood culture bottle and generally require an additional 1 to 3 days before organism identification. Due to the fact that bacterial DNA can be obtained directly by extraction from positive blood culture bottles for the PCR step of TRF profiling, significant delays that result from the need for isolated colonies for biochemical identification methods are avoided. Also, TRF profiling for bacterial identification is more rapid than sequencing of the $16 \mathrm{~S}$ rRNA gene, primarily because it does not require a second thermal cycler reaction to produce dye terminated products for sequencing. The analysis of $16 \mathrm{~S}$ rRNA genes by TRF profiling allows the identification process to be completed within $\sim 8$ hours after obtaining a positive blood bottle sample. Reduced diagnostic time has implications for duration of infection, the cost of patient care, length of hospitalization, development of broad-spectrum antibiotic resistance, and mortality due to bacteremia. TRF profiling represents a highly reproducible and predictive source for identification of many organisms associated with bacteremia.

\section{ACKNOWLEDGMENTS}

We thank the people at Marshfield Laboratories for provision of blood cultures and isolates for analysis. We also thank Marshfield Clinic Research Foundation for its support through the assistance of Alice Stargardt in the preparation of this manuscript.

\section{REFERENCES}

1. Elting LS, Rubenstein EB, Rolston KV, Bodey GP. Outcomes of bacteremia in patients with cancer and neutropenia: observations from two decades of epidemiological and clinical trials. Clin Infect Dis 1997;25:247-259.

2. Jarvis WR. Selected aspects of the socioeconomic impact of nosocomial infections: morbidity, mortality, cost, and prevention. Infect Control Hosp Epidemiol 1996;17:552-557.

3. Weinstein MP, Towns ML, Quartey SM, Mirrett S, Reimer LG, Parmigiani G, Reller LB. The clinical significance of positive blood cultures in the 1990s: a prospective comprehensive evaluation of the microbiology, epidemiology, and outcome of bacteremia and fungemia in adults. Clin Infect Dis 1997;24:584-602.

4. Rezende NA, Blumberg HM, Metzger BS, Larsen NM, Ray SM, McGowan JE Jr. Risk factors for methicillin-resistance among patients with Staphylococcus aureus bacteremia at the time of hospital admission. Am J Med Sci 2002;323:117-123.

5. Roghmann MC. Predicting methicillin resistance and the effect of inadequate empiric therapy on survival in patients with Staphylococcus aureus bacteremia. Arch Intern Med 2000;160:1001-1004.

6. Chassy BM, Murphy CM. Lactococcus and Lactobacillus. In: Sonenshein AL, Hoch JA, Losick R, eds. Bacillus subtilis and other gram-positive bacteria: biochemistry, physiology, and molecular genetics. Washington DC: American Society for Microbiology; 1993.

7. Husni RN, Gordon SM, Washington JA, Longworth DL. Lactobacillus bacteremia and endocarditis: review of 45 cases. Clin Infect Dis 1997;25:1048-1055.

8. Antony SJ, Stratton CW, Dummer JS. Lactobacillus bacteremia: description of the clinical course in adult patients without endocarditis. Clin Infect Dis 1996;23:773-778.

9. Bayer AS, Chow AW, Betts D, Guze LB. Lactobacillemia--report of nine cases. Important clinical and therapeutic considerations. Am J Med 1978;64:808-813.

10. Biocca E, Seppilli A. Human infections caused by lactobacilli. J Infect Dis 1942;84:112-115.

11. Schlegel L, Lemerle S, Geslin P. Lactobacillus species as opportunistic pathogens in immunocompromised patients. Eur J Clin Microbiol Infect Dis 1998;17:887-888.

12. Cox SM, Phillips LE, Mercer LJ, Stager CE, Waller S, Faro S. Lactobacillemia of amniotic fluid origin. Obstet Gynecol 1986;68:134-135.

13. Bantar CE, Relloso S, Castell FR, Smayevsky J, Bianchini HM. Abscess caused by vancomycin-resistant Lactobacillus confusus. J Clin Microbiol 1991;29:2063-2064.

14. Brouqui P, Raoult D. Endocarditis due to rare and fastidious bacteria. Clin Microbiol Rev 2001;14:177-207.

15. Cooper CD, Vincent A, Greene JN, Sandin RL, Cobian L. Lactobacillus bacteremia in febrile neutropenic patients in a cancer hospital. Clin Infect Dis 1998;26:1247-1248.

16. Fruchart C, Salah A, Gray C, Martin E, Stamatoullas A, Bonmarchand G, Lemeland JF, Tilly H. Lactobacillus species as emerging pathogens in neutropenic patients. Eur J Clin Microbiol Infect Dis 1997;16:681-684.

17. Felten A, Barreau C, Bizet C, Lagrange PH, Philippon A. Lactobacillus species identification, $\mathrm{H}_{2} \mathrm{O}_{2}$ production, and antibiotic resistance and correlation with human clinical status. J Clin Microbiol 1999;37:729-733.

18. Christensen JE, Stencil JA, Reed KD. Rapid identification of bacteria from positive blood cultures by terminal restriction fragment length polymorphism profile analysis of the 16S rRNA gene. J Clin Microbiol 2003;41:3790-3800.

19. Kandler O, Weiss N. Regular, nonsporing Gram-positive rods. In: Sneath PHA, Mair NS, Sharpe ME, Holt JG, eds. Bergey's manual of systematic bacteriology. Baltimore, MD: Williams \& Wilkins; 1986. 1208-1234. 
20. Altschul SF, Gish W, Miller W, Myers EW, Lipman DJ. Basic local alignment search tool. J Mol Biol 1990;215:403-410.

21. Cole JR, Chai B, Marsh TL, Farris RJ, Wang Q, Kulam SA, Chandra S, McGarrell DM, Schmidt TM, Garrity GM, Tiedje JM; Ribosomal Database project. The Ribosomal Database Project (RDP-II): previewing a new autoaligner that allows regular updates and the new prokaryotic taxonomy. Nucleic Acids Res 2003;31:442-443.

22. Thompson JD, Higgins DG, Gibson TJ. CLUSTAL W:

improving the sensitivity of progressive multiple sequence alignment through sequence weighting, position-specific gap penalties and weight matrix choice. Nucleic Acids Res 1994;22:4673-4680.

23. Southern EM. Measurement of DNA length by gel electrophoresis. Anal Biochem 1979;100:319-323. 